\title{
AN OBSERVATIONAL LIMIT TO THE EVOLUTIONARY TIME SCALE OF THE 13,000 K WHITE DWARF G117-B15A
}

\author{
S. O. KEPLER \\ Instituto de Fisica, Universidade Federal do Rio Grande do Sul, Brazil \\ G. Vauclair, N. Dolez, ANd M. Chevreton \\ Observatoire Midi-Pyrenees, France \\ M. A. BARSTOW \\ Department of Physics and Astronomy, University of Leicester \\ R. E. Nather, D. E. Winget, J. L. Provencal, and J. C. Clemens \\ McDonald Observatory and Department of Astronomy, University of Texas at Austin \\ AND \\ G. FontaIne \\ Département de Physique, Université de Montréal \\ Received 1989 September 1; accepted 1989 December 4
}

\begin{abstract}
Nature has provided us with a way of measuring the evolutionary time scale of some stars through asteroseismology, i.e., the study of stellar interiors through their intrinsic variations in brightness. By measuring the slow, secular change in one or more of its periodicities, we can measure the rate at which a luminosityvariable white dwarf is cooling.

Using $204 \mathrm{hr}$ of high-speed photometric observations of the DAV star G117-B15A during the last $14 \mathrm{yr}$, we have obtained an observational limit to the rate of period change for its dominant pulsation at $215.2 \mathrm{~s}$ of $d P / d t=(8.3 \pm 5.0) \times 10^{-15} \mathrm{~s} \mathrm{~s}^{-1}$. This rate of period change corresponds to an evolutionary time scale of $\tau=(8.2 \pm 5.0) \times 10^{8} \mathrm{yr}$, consistent with the change expected due to cooling of the white dwarf undergoing nonradial $g$-mode pulsations. The observed limit on the rate of period change makes G117-B15A the most accurate optical clock known, with a stability comparable to that of the atomic clocks used by the National Bureau of Standards, and exceeded only by a few ms radio pulsars.

Since all DA white dwarfs are thought to cool through the DAV instability strip, and there are no known differences between the properties of variables and nonvariable white dwarfs, the measurement of an evolutionary time scale, suitably scaled by mass and surface temperature, should apply to all DA white dwarfs.

Subject headings: stars: evolution — stars: individual (G117-B15A) - stars: pulsation — stars: white dwarfs
\end{abstract}

\section{INTRODUCTION}

The DAV stars are the ZZ Ceti white dwarfs which show luminosity variations; except for their variability, the DAV stars are completely normal DA white dwarfs. Fontaine et al. (1982) and Greenstein (1982), using homogeneous sets of colors, found that all observed DA white dwarfs in the color range corresponding to effective temperatures in a narrow strip ( $\sim 1400 \mathrm{~K}$ wide) around $11,000 \mathrm{~K}$ were $\mathrm{ZZ}$ Ceti variables. Similar results were obtained from IUE spectra (Wesemael, Lamontagne, and Fontaine 1986).

There are 20 known DAV stars, all multiperiodic, with periods ranging from 109 to $1186 \mathrm{~s}$ and peak-to-peak light variations from $2 \%-34 \%$. The observed variations are due to nonradial $g$-mode pulsations (Kepler 1984) and, since each pulsation mode constrains independently the properties and structure of the star, the observed pulsations can be used to infer the internal structure of the white dwarf, as demonstrated by the mode trapping calculations of Winget, Van Horn, and Hansen (1981). The white dwarf structure is chemically stratified, with the lighter elements floating on top of the heavier ones, due to gravitational settling. The study of their pulsations therefore is also a probe of diffusion theory. Asteroseismology of prewhite dwarfs have allowed us to measure for the first time the secular cooling time scale for the star PG 1159-035
(Winget et al. 1985), and to deduce its mass to unprecedented accuracy (Kawaler 1989).

The study of white dwarf pulsations also provides a unique insight into the physics of matter at extreme conditions of temperature and pressure, inaccessible in terrestrial laboratories, such as the rate of energy transport by various neutrino processes, and can provide contraints on the presence or absence of low mass axions. As pointed out by McGraw (1979), the DAV stars are found near the hydrogen opacity maximum, indicating that the pulsations are associated with the development of partial ionization zones in the surface layers (Dolez and Vauclair 1981; Winget et al. 1982). Thus the DAV variability is a consequence of normal evolution of a DA star into the temperature region where a significant partial ionization zone develops. For a review of the properties of the DAV stars and other compact pulsators, see Winget (1988).

Observations of these compact pulsators with the Whole Earth Telescope (WET) have shown that we can identify a large number of pulsation modes from the observed light curve and finally start to measure the stellar structural properties (Nather 1989; Winget et al. 1990).

The pulsation periods of the DAV stars change with time due to secular changes in their internal structures. The time scale for period change is directly proportional to the cooling 
time scale of the white dwarf, and its value is sensitive to the chemical composition of the white dwarf's core material. In principle, observed rates of secular cooling can be used to distinguish among white dwarfs of differing core composition.

Evolutionary time scale measurements are also critical to the method of directly measuring the age of the galactic disk by measuring the age of the coolest white dwarfs. The first white dwarfs formed on the disk of our Galaxy are still visible, and therefore the white dwarf luminosity function can be used to measure the age of the Galactic disk (Winget et al. 1987).

\section{OBSERVATIONS}

G117-B15A was shown to pulsate by McGraw and Robinson (1976) and is one of the DAV stars with the simplest light variations. Kepler et al. (1982) observed G117-B15A with highspeed photometry for $85 \mathrm{hr}$ to decipher its light curve, and found that it is dominated by a pulsation with a period of $215.2 \mathrm{~s}$ and an amplitude of $22 \mathrm{mmag}$, but that it also shows five other periodicities with smaller amplitudes. In 1982 we derived an upper limit for the rate of change of period with time of $|\dot{P}| \leq 7.8 \times 10^{-14} \mathrm{~s} \mathrm{~s}^{-1}$ for this pulsation mode.
Since 1982 we have added a total of $119 \mathrm{hr}$ of high-speed photometry to our measurements of the star. A total of $78 \mathrm{hr}$ were obtained using a two-star photometer (Nather 1973) on the $2.1 \mathrm{~m}$ Struve telescope and $4 \mathrm{hr}$ on the $91 \mathrm{~cm}$ telescope at McDonald Observatory, $1.8 \mathrm{hr}$ on the $2.5 \mathrm{~m}$ Isaac Newton telescope at La Palma, and $0.6 \mathrm{hr}$ on the CFHT $3.6 \mathrm{~m}$ at Mauna Kea. We also obtained $25 \mathrm{hr}$ of data on the $1.9 \mathrm{~m}$ telescope at Haute Provence Observatory with a three-channel photometer (Vauclair et al. 1989). The total data set now spans 14 years.

The observations were obtained with blue-sensitive bi-alkali phototubes, and as G117-B15A is a rather faint, $V=15.52$ (Eggen and Greenstein 1965), and since the light variations are due exclusively to temperature variations, and therefore in phase at different wavelengths (Kepler 1984), all measurements were obtained in unfiltered light in order to improve the photon detection rate. The amplitudes observed should be used with caution, since they are wavelength dependent (Robinson, Kepler, and Nather 1982; Brassard, Wesemael, and Fontaine 1987), and the effective wavelength of our white-light photometry is not well determined. Table 1 gives a journal of

TABLE 1

JOURNAL OF OBSERVATIONS SINCE 1982

\begin{tabular}{|c|c|c|c|c|c|}
\hline Run & $\begin{array}{l}\text { Length of } \\
\text { Observation } \\
\text { (hr) }\end{array}$ & $\begin{array}{l}\text { Integration } \\
\text { Time } \\
\text { (s) }\end{array}$ & $\begin{array}{c}\text { Fractional } \\
\text { Semiamplitude }\end{array}$ & $\begin{array}{c}\text { BJDD at } \\
\text { Maximum } \\
(2440000.0+)\end{array}$ & $\begin{array}{c}\text { Error } \\
\text { (s) }\end{array}$ \\
\hline 2593 & 0.9 & 10 & 0.024 & 4637.776174 & 1.6 \\
\hline 2597 . & 7.0 & 10 & 0.023 & 4641.624287 & 0.5 \\
\hline 2629 & 2.1 & 10 & 0.022 & 4992.789531 & 1.2 \\
\hline 2633 . & 6.6 & 10 & 0.021 & 4994.689956 & 0.7 \\
\hline $2637 \ldots \ldots \ldots \ldots \ldots \ldots \ldots \ldots$ & 3.4 & 10 & 0.024 & 4996.744801 & 0.8 \\
\hline 2640 . & 3.7 & 10 & 0.024 & 4997.723649 & 0.7 \\
\hline JES1 $\ldots$ & 2.9 & 10 & 0.021 & 5021.716661 & 1.0 \\
\hline $2869 \ldots$ & 3.2 & 10 & 0.022 & 5703.860004 & 1.4 \\
\hline $2870 \ldots$ & 5.6 & 10 & 0.028 & 5734.642701 & 0.7 \\
\hline 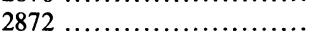 & 2.4 & 10 & 0.025 & 5735.643972 & 0.8 \\
\hline ER60. & 3.8 & 5 & 0.023 & 6113.763716 & 0.7 \\
\hline ER110 & 1.8 & 5 & 0.021 & 6443.775386 & 1.0 \\
\hline 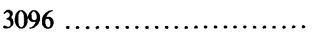 & 5.9 & 5 & 0.023 & 6468.630178 & 0.5 \\
\hline $3106 \ldots \ldots \ldots \ldots \ldots \ldots \ldots \ldots$ & 1.2 & 10 & 0.024 & 6473.718679 & 0.9 \\
\hline 3141 … & 1.0 & 10 & 0.022 & 6523.620086 & 1.3 \\
\hline 3143 . & 1.0 & 10 & 0.020 & 6524.613917 & 1.3 \\
\hline AT13 $^{\mathbf{a}} \ldots \ldots \ldots \ldots \ldots \ldots \ldots$ & 3.8 & 5 & 0.018 & 6768.855451 & 2.3 \\
\hline $9004 \ldots \ldots \ldots \ldots \ldots \ldots \ldots \ldots$ & 2.0 & 10 & 0.022 & 6794.935676 & 1.0 \\
\hline 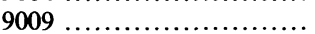 & 1.2 & 10 & 0.018 & 6796.928219 & 1.9 \\
\hline $9012 \ldots \ldots \ldots \ldots \ldots \ldots \ldots \ldots$ & 1.9 & 10 & 0.022 & 6797.924535 & 1.3 \\
\hline 9014 . … $\ldots \ldots \ldots \ldots \ldots \ldots \ldots$ & 2.8 & 10 & 0.024 & 6798.903378 & 0.9 \\
\hline DEW1 …................. & 1.7 & 10 & 0.018 & 6823.663537 & 1.5 \\
\hline DEW2 … $\ldots \ldots \ldots \ldots \ldots$ & 4.5 & 10 & 0.020 & 6825.651132 & 1.6 \\
\hline GV1 … & 2.4 & 6 & 0.022 & 7231.328096 & 1.1 \\
\hline REN23 ..... & 3.0 & 10 & 0.022 & 7231.612054 & 0.7 \\
\hline GV2 ......... & 1.5 & 6 & 0.017 & 7232.396626 & 3.4 \\
\hline REN25 & 2.4 & 10 & 0.020 & 7232.623291 & 2.7 \\
\hline 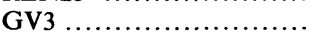 & 2.9 & 6 & 0.019 & 7233.343090 & 2.1 \\
\hline REN27 & 2.2 & 10 & 0.022 & 7233.634506 & 1.9 \\
\hline GV4 ... & 3.4 & 6 & 0.022 & 7234.319475 & 1.3 \\
\hline GV5 ….................. & 3.2 & 6 & 0.020 & 7235.313250 & 1.6 \\
\hline REN30 ................... & 2.7 & 10 & 0.021 & 7235.607168 & 1.2 \\
\hline REN32 …................ & 2.5 & 10 & 0.022 & 7236.610922 & 0.9 \\
\hline JCC69 … $\ldots \ldots \ldots \ldots \ldots \ldots$ & 0.6 & 5 & 0.019 & 7499.072036 & 3.0 \\
\hline …............ & 6.1 & 10 & 0.021 & 7532.768799 & 1.0 \\
\hline REN60 .................. & 1.8 & 10 & 0.020 & 7589.375198 & 1.0 \\
\hline GV41 …............... & 4.1 & 10 & 0.020 & 7594.331735 & 1.3 \\
\hline GV43 ….............. & 1.8 & 10 & 0.019 & 7595.323018 & 1.7 \\
\hline 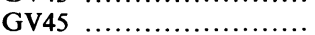 & 2.0 & 10 & 0.020 & 7596.311907 & 2.1 \\
\hline GV47 …................ & 2.0 & 10 & 0.023 & 7597.315602 & 1.4 \\
\hline GV49. & 2.0 & 10 & 0.019 & 7598.319339 & 2.9 \\
\hline
\end{tabular}

${ }^{\text {a }}$ Data taken on the $91 \mathrm{~cm}$ telescope, with companion inside aperture. 
the observations since 1982 . The quoted errors throughout this paper are $1 \sigma$.

From these and the earlier measurements we derive a value $d P / d t=(8.3 \pm 5.0) \times 10^{-15} \mathrm{~s} \mathrm{~s}^{-1}$, equivalent to a time scale for period change of $P / \dot{P}=(8.2 \pm 5.0) \times 10^{8}$ yr. This value, which we take as a limit, is only slightly larger than the theoretical value for the cooling time scale of a DA white dwarf around $13,000 \mathrm{~K}$ (Winget and van Horn 1987), the temperature of G117-B15A (Wesemael et al. 1986).

\section{DATA ANALYSIS}

The method for data reduction and conversion of the time base from UTC, received at the observatories from broadcast time signals, to the more uniform Barycentric Julian Dynamical Date (previously called the Barycentric Julian Ephemeris Date) was described in Kepler et al. (1982). The method takes in account all the leap seconds introduced into UTC, as well as the difference in light travel time due to the motion of the Earth around the barycenter of the solar system, to an accuracy better than $0.3 \mathrm{~s}$.

The first step of our analysis was to take Fourier transforms of each night's data to verify which pulsations were present. We had to take in account that only in runs longer than $\sim 4 \mathrm{hr}$ could we see the three largest amplitude pulsations separately, and that the three smallest amplitude pulsations could only be seen in power spectra combining all the data from a single observing season. We conclude from a detailed examination of the power spectra that the pulsational properties of G117B15A have remained unchanged during the 14 years we have observed the star; the six pulsation modes detected in 1982 are still present in the data, with the $215.2 \mathrm{~s}$ pulsation dominating the light curve.

\section{THE UPPER LIMIT TO $\dot{P}$}

Once we had assured ourselves of the pulsational stability of the star, we could procede with the analysis. We fit each night's data with a sinusoid with a period of $215.2 \mathrm{~s}$ by least squares, to obtain a mean estimate of the time of pulsation maximum for that date. After correcting the times of maxima to the barycenter of the solar system, we calculated the difference between the observed $(O)$ times of maxima and the times of maxima calculated $(C)$ using a linear ephemeris starting on our first observation, obtaining therefore an $(O-C)$ diagram (Fig. 1). Unique cycle counts $E$ were obtained for each night, since the period derived in 1982 was sufficiently accurate for us to bridge the whole data base without any possibility of cycle count error. The fairly large apparent scatter in the diagram is an artifact of the varying quality of the different runs; no data point lies more than $3 \sigma$ away from zero.

The rate of period change for the pulsation, as well as a correction to the period and epoch, can then be obtained by fitting a parabola to the $(O-C)$; i.e., we assume a fit of the form $C=E_{0}+P \times E$ and what we obtain from the fit are corrections to the initial values of $P$ and $E_{0}$, which we call $\Delta E_{0}$ and $\Delta P$ :

$$
(O-C)=\Delta E_{0}+\Delta P \times E+\frac{1}{2} P \times \dot{P} \times E^{2},
$$

where $\Delta E_{0}$ is the correction to the epoch of observation, $\Delta P$ is the correction to the period, $E$ is the number of cycles elapsed since $E_{0}$, and $\dot{P}$ is the rate of change of period with time, $d P / d t$.

Using this formula to fit a parabola by weighted leastsquares to the 75 times of maxima obtained since 1975, one for

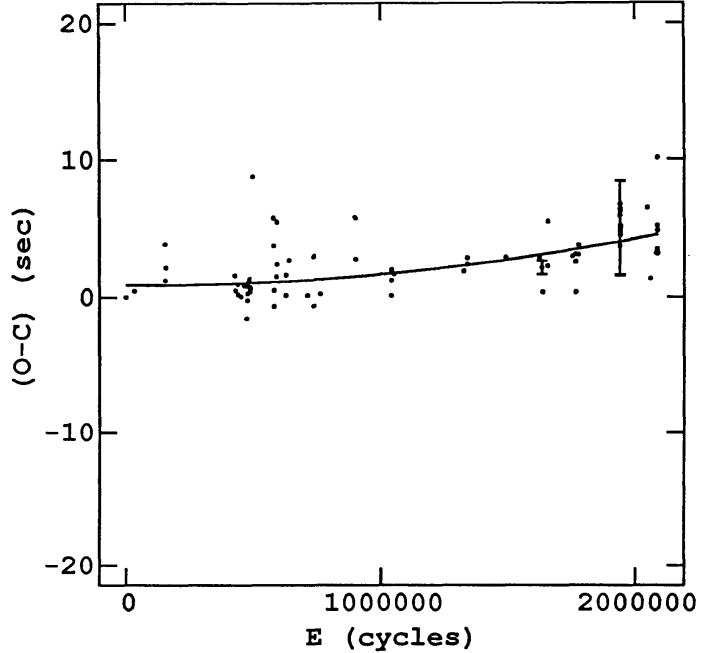

FIG. 1.-The $(O-C)$ diagram for the $215.2 \mathrm{~s}$ pulsation of G117-B15A. The observed times of maxima are listed in Table 1, and the calculated times of maxima were derived using a linear ephemeris starting on BJED 2442397.917520 . For clarity, we have included only the smallest and largest error bars.

each run of data, we obtain:

$$
\begin{aligned}
E_{0} & =\text { BJDD } 2442397.917522 \pm 0.000007 \text { days } \\
P & =215.1973878 \pm 0.0000013 \mathrm{~s} \\
d P / d t & =(8.3 \pm 5.0) \times 10^{-15} \mathrm{~s} \mathrm{~s}^{-1}
\end{aligned}
$$

or

$$
P / \dot{P}=(8.2 \pm 5.0) \times 10^{8} \mathrm{yr} .
$$

We consider the observed $\dot{P}$ only as an upper limit, since its error is less than $2 \sigma$ smaller. Table 2 gives the evolution of our measurement, i.e., the results of the parabolic fit to the $(\mathrm{O}-\mathrm{C})$ values, including data obtained from 1975 up to that year.

\section{DISCUSSION}

A measured rate of change of period of order $10^{-14} \mathrm{~s} \mathrm{~s}^{-1}$, like the limit we observe for G117-B15A, places a very stringent restriction on the dynamical environment of the white dwarf, since even a planet of the mass of Jupiter, and at the same distance from the white dwarf that Jupiter is from the Sun, would give an apparent rate of change of the pulsation period, i.e., of the arrival time of the pulses of the order of $10^{-12} \mathrm{~s} \mathrm{~s}^{-1}$, with the times of maxima varying periodically as the pulsating star executes its reflex motion around the center of mass of the system. Therefore, to have a $\dot{P}$ of the order of $10^{-14} \mathrm{~s} \mathrm{~s}^{-1}$, the white dwarf cannot have any body of even a

TABLE 2

Evolution OF THE $\dot{P}$ Measurement

\begin{tabular}{cr}
\hline \hline Year & \multicolumn{1}{c}{$d P / d t$} \\
\hline $1980 \ldots \ldots \ldots \ldots \ldots$ & $(5.5 \pm 4.7) \times 10^{-14}$ \\
$1981 \ldots \ldots \ldots \ldots \ldots \ldots$ & $(5.3 \pm 2.3) \times 10^{-14}$ \\
$1982 \ldots \ldots \ldots \ldots \ldots \ldots$ & $(0.4 \pm 2.2) \times 10^{-14}$ \\
$1984 \ldots \ldots \ldots \ldots \ldots \ldots$ & $(0.5 \pm 1.3) \times 10^{-14}$ \\
$1985 \ldots \ldots \ldots \ldots \ldots \ldots$ & $(0.4 \pm 1.1) \times 10^{-14}$ \\
$1986 \ldots \ldots \ldots \ldots \ldots$ & $-(0.6 \pm 0.8) \times 10^{-14}$ \\
$1987 \ldots \ldots \ldots \ldots \ldots$ & $-(0.4 \pm 0.7) \times 10^{-14}$ \\
$1988 \ldots \ldots \ldots \ldots \ldots$ & $(1.2 \pm 0.6) \times 10^{-14}$ \\
$1989 \ldots \ldots \ldots \ldots \ldots$ & $(0.8 \pm 0.5) \times 10^{-14}$ \\
\hline
\end{tabular}


modest planetary mass orbiting it. This precision time-lag technique of measurement may therefore prove itself ultimately capable of detecting planets outside the solar system, in addition to producing information on the stellar structure and evolution of the primary target star (Kepler et al. 1988).

The most interesting aspect of our new limit on the rate of period change is how it compares to the theoretical rate of period change of nonradial $g$-mode pulsations caused by the cooling of the white dwarf.

We can show that the observed modes have low $l$ values by simple geometrical considerations: increasing $l$ significantly increases the cancellation of bright and dark regions of the star leading to (currently) unobservable amplitudes for $l \gtrsim 4$ (Dziembowski 1977). Given a low value of $l$, the observed period assures us that the pulsation has also a low $k$ value.

For low $l$, low $k$ modes in evolutionary $\mathrm{C} / \mathrm{O}$ core DAV models appropriate to G117-B15A ( $\left.T_{\text {eff }} \sim 13,000 \mathrm{~K}\right)$, we find $\dot{P} \approx 3 \times 10^{-15} \mathrm{~s} \mathrm{~s}^{-1}$, or $P / \dot{P} \approx 2 \times 10^{9}$ yr (Winget 1981 ; Wood and Winget 1989; Bradley, Winget, and Wood 1989). The precise value is a function of $k, l$, and the physical parameters of the star-particularly core composition-but is essentially given by $\tau=P / \dot{P}=a T / T$, where the coefficient $a$ is of order unity (see Winget 1988).

Although our limit on $\dot{P}$ is still above the value expected for a $\mathrm{C} / \mathrm{O}$ core, we can already use it to eliminate any core composition significantly heavier than $\mathrm{Ne} / \mathrm{Mg}$. The straightforward reduction of the cooling times by the ratio of the mean molecular weights, as in the simple theory of Mestel (1952), can be dwarfed by the effect of Debye cooling. Any compositions containing elements substantially heavier than $\mathrm{Ne} / \mathrm{Mg}$ will crystallize at temperatures well above the DAV (ZZ Ceti) instability strip (Lamb and Van Horn 1975). This implies that the cooling rate will be much faster than inferred from the Mestel theory (Winget and Van Horn 1987) and is therefore ruled out by our observational limit.

Since our limit on $P / \dot{P}$ will continue to improve quadratically with the time baseline over which the star is observed, or $\sim 17 \%$ per year currently, we expect to reach the predicted time scale for a $\mathrm{C} / \mathrm{O}$ core in $10 \mathrm{yr}$. If, on the other hand, the value for $\dot{P}$ we obtained is real, in $\sim 4$ yr it will become a $3 \sigma$ measurement. This might imply that this star has a core composition more along the lines of $\mathrm{O} / \mathrm{Ne} / \mathrm{Mg}-$ a particularly interesting possibility in view of the detection of these elements in the ejecta of classical novae (Ferland and Shields 1978; Williams et al. 1985). It is interesting to note that cores rich in $\mathrm{Ne}$ and $\mathrm{Mg}$ will crystallize right at the blue edge of the DAV instability strip. Thus if G117-B15A has such a core composition it should be crystallizing even as we watch.

In either case we will measure the evolutionary time scale for a cool white dwarf, equivalent to that measured by Winget et al. (1985) for the hot white dwarf PG1159-035, on a time scale somewhat shorter than a human lifetime.

We are grateful to Edward L. Robinson, Jan-Erik Solheim, and Austin Tomaney for acquiring some of the data referred to in this paper. This work was partially supported by grants from CNPq-Brazil, NSF-USA, CNRS-France, SERC-UK and NSERC-Canada.

Bradley, P. A., Winget, D. E., and Wood, M. A. 1989, in Proc. of the IAU Colloquium 114, White Dwarfs, ed. G. Wegner (Berlin: Springer), p. 286.

Brassard, P., Wesemael, F., and Fontaine, G. 1987, in Proc. of the IAU Colloquium 95, The Second Conference on Faint Blue Stars, ed. A. G. D. Philip, D. S. Hayes, and J. W. Liebert (Schenectady: L. Davis Press), p. 669

Dolez, N., and Vauclair, G. 1981, Astr. Ap., 102, 375.

Dziembowski, W. 1977, Acta Astr. 27, 203.

Eggen, O. J., and Greenstein, J. L. 1965, Ap. J., 141, 183.

Ferland, G. J., and Shields, G. A. 1978, Ap. J., 226, 172.

Fontaine, G., McGraw, J. T., Dearborn, D. S. P., Gustafson, J., and Lacombe, P. 1982, Ap. J., 258, 651 .

Greenstein, J. L. 1982, Ap. J., 258, 661

Kawaler, S. D. 1989, in Proc. of the IAU Colloquium 114, White Dwarfs, ed. G. Wegner (Berlin: Springer), p. 97.

Kepler, S. O. 1984, Ap. J., 286, 314

Kepler, S. O., Robinson, E. L., Nather, R. E., and McGraw, J. T. 1982, Ap. J., 254, 676.

Kepler, S. O., Winget, D. E., Robinson, E. L., and Nather, R. E. 1988, in $I A U$ Symposium 123, Advances in Helio- and Asteroseismology, ed. J. ChristensenDalsgaard and S. Frandsen (Dordrecht: Reidel), p. 325.

Lamb, D. Q., and Van Horn, H. M. 1975, Ap. J. 200, 306.

McGraw, J. T. 1979, Ap. J., 229, 203.

McGraw, J. T., and Robinson, E. L. 1976, Ap. J. (Letters), 205, L155.

Mestel, L. 1952, M.N.R.A.S., 112, 583.

Nather, R. E. 1973, Vistas in Astronomy, 15, 91.

\section{REFERENCES}

Nather, R. E. 1989, in Proc. of the IAU Colloquium 114, White Dwarfs, ed. G. Wegner (Berlin: Springer), p. 109

Robinson, E. L., Kepler, S. O., and Nather, R. E. 1982, Ap. J., 259, 219

Vauclair, G., Goupil, M. J., Baglin, A., Auvergne, M., and Chevreton, M. 1989 Astr. Ap., 215, L17.

Wesemael, F., Lamontagne, R., and Fontaine, G. 1986, A.J., 91, 1376.

Williams, R. E., Ney, E. P., Sparks, W. M., Starrfield, S., and Truran, J. W. 1985, M.N.R.A.S., 212, 753.

Winget, D. E. 1981, Ph.D thesis, University of Rochester.

. 1988, in IAU Symposium 123, Advances in Helio- and Asteroseismology, ed. J. Christensen-Dalsgaard and S. Frandsen (Dordrecht: Reidel), p. 305

Winget, D. E., et al. 1990, Ap. J., in press.

Winget, D. E., Hansen, C. J., Liebert, J., Van Horn, H. M., Fontaine, G., Nather, R. E., Kepler, S. O., and Lamb, D. Q. 1987, Ap. J. (Letters), 315, L77.

Winget, D. E., Kepler, S. O., Robinson, E. L., Nather, R. E., and O'Donoghue, D. $1985, A p . J .292,606$

Winget, D. E., and Van Horn, H. M. 1987, in Proc. of the IAU Colloquium 95 The Second Conference on Faint Blue Stars, ed. A. G. D. Philip, D. S. Hayes, and J. W. Liebert (Schenectady: L. Davis Press), p. 368.

Winget, D. E., Van Horn, H. M., and Hansen, C. J. 1981, Ap. J. (Letters), 245, L33.

Winget, D. E., Van Horn, H. M., Tassoul, M., Hansen, C. J., Fontaine, G., and Carrol, B. W. 1982, Ap. J. (Letters), 252, L65.

Wood, M. A., and Winget, D. E. 1989, in Proc. of the IAU Colloquium 114 White Dwarfs, ed. G. Wegner (Berlin: Springer), p. 282.

M. A. BARSTOw: Department of Physics and Astronomy, University of Leicester, University Road, Leicester LE1 7RH, UK

M. Chevreton: Observatoire de Paris-Meudon CNRS UA 173 F-92195, Meudon Cedex, France

N. Dolez: C.E.R.F.A.C.S., Avenue G Coriolis, 31100, Toulouse, France

G. Fontaine: Département de Physique, Université de Montréal, P.O. Box 6128, Succ. A, Montréal, P.Q. Canada, H3C 3J7

S. O. KePLER: Instituto de Fisica da UFRGS, 91500 Porto Alegre, RS, Brazil

R. E. Nather, D. E. Winget, J. L. Provencal, and J. C. Clemens: Department of Astronomy, The University of Texas, Austin, TX 78712-1083

G. VAUCLAIR: Observatoire Midi-Pyrenees CNRS UA 285, 14, Avenue Edouard Belin, 31400 Toulouse, France 